\title{
Conference Paper \\ Optimal Control of Particle Advection in Couette and Poiseuille Flows
}

\author{
Teresa Grilo, ${ }^{1,2}$ Fernando Lobo Pereira, ${ }^{1}$ and Sílvio Gama ${ }^{2}$ \\ ${ }^{1}$ Instituto de Sistemas e Robótica, FEUP, Rua Roberto Frias s/n, 4200-465 Porto, Portugal \\ ${ }^{2}$ Centro de Matemática, FCUP, Rua do Campo Alegre, No. 687, 4169-007 Porto, Portugal
}

Correspondence should be addressed to Teresa Grilo; tgrilo@fc.up.pt

Received 16 June 2013; Accepted 30 July 2013

Academic Editors: G. S. F. Frederico, N. Martins, D. F. M. Torres, and A. J. Zaslavski

This Conference Paper is based on a presentation given by Teresa Grilo at "The Cape Verde International Days on Mathematics 2013" held from 22 April 2013 to 25 April 2013 in Praia, Cape Verde.

Copyright (C) 2013 Teresa Grilo et al. This is an open access article distributed under the Creative Commons Attribution License, which permits unrestricted use, distribution, and reproduction in any medium, provided the original work is properly cited.

\begin{abstract}
We present the problem of minimum time control of a particle advected in Couette and Poiseuille flows and solve it by using the Pontryagin maximum principle. This study is a first step of an effort aiming at the development of a mathematical framework for the control and optimization of dynamic control systems whose state variable is driven by interacting ODEs and PDEs which can be applied in the control of underwater gliders and mechanical fishes.
\end{abstract}

\section{Introduction}

This paper represents a first step for the optimal control of dynamic systems whose state evolves through the interaction of ordinary differential equations and the partial differential equations, $[1,2]$, which will provide a sound basis for the design and control of new advanced engineering systems. In Figure 1, two representative examples of the class of applications are considered: (i) underwater gliders, that is, winged autonomous underwater vehicles (AUVs) which locomote by modulating their buoyancy and their attitude in its environment, and (ii) robotic fishes. Motion modeling of these two types of systems can be found in $[3,4]$ and [5], respectively.

In spite of the key roots of the Optimal Control Theory having been established in the sixties for control systems with dynamics given by ordinary differential equations, [6], its sophistication in multiple directions has been progressing unabated (see, among others, $[7,8]$ ). However, there still remains a large gap in what concerns dynamic control systems driven by partial differential equations, [2], and it is largely inexistent for hybrid systems in the sense that the controlled dynamics involve both partial and ordinary differential equations.
In this paper, we formulate and solve two optimal control problems. Each one of these problems corresponds to a particular solution of the incompressible Navier-Stokes equation in two spatial dimensions. These particular solutions are, respectively, the steady Couette and Poiseuille flows.

The Couette flow is the steady laminar unidirectional and two-dimensional flow due to the relative motion of two infinite horizontal and parallel rigid plates [9]. The liquid between these two plates is driven by the viscous drag force originated by the uniform motion of the upper plate which moves in the $x$-direction with velocity $v_{0}$ (the lower plate is at rest). In this case, the velocity of such a flow has a linear profile and is given by

$$
v(x, y)=(m y, 0), \quad x \in \mathbb{R}, y \in[0, L]
$$

with $m=v_{0} / L$, the plates being $L$ distance units apart (Figure 2(a)).

The Poiseuille flow is the steady flow due to the presence of a pressure gradient between two fixed (i.e., with zero relative velocity) rigid plates [9]. In this case, a parabolic velocity profile is of the form

$$
v(x, y)=\left(a-\frac{a}{L^{2}} y^{2}, 0\right), \quad x \in \mathbb{R}, y \in[-L, L],
$$




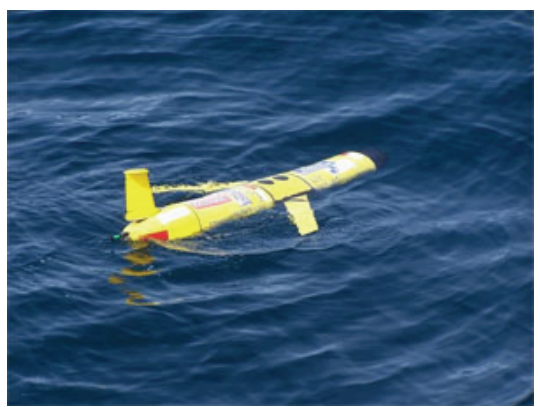

(a)

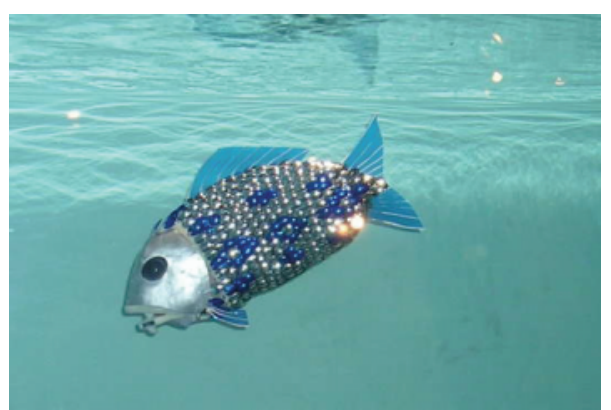

(b)

FIGURE 1: Underwater glider (a), robotic fish (b).

where, now, $L$ is half the distance between the upper and lower plates (Figure 2(b)).

\section{Minimum Time Control Problem}

Consider a flow in a channel of the width $L$ with a given velocity field and a particle placed in this flow. Let $(0, b)$ be the initial position of the particle, with $0 \leq b \leq L$.

The objective of this problem is to determine the control function $u(\cdot)=\left(u_{x}(\cdot), u_{y}(\cdot)\right)$ to be applied to the particle so that it will move in the channel from the initial position to the end point $\left(x_{f}, b\right)$ in minimum time while subject to the flow field. Let $X(t)=(x(t), y(t))$ be the position of the particle at time $t$, the control problem can be formulated as follows:

$$
\begin{aligned}
\text { Minimize } & T \\
\text { subject to } & \dot{X}(t)=F(X(t), u(t)), \\
& X(0)=(0, b), \\
& X(T)=\left(x_{f}, b\right), \quad \forall t \in[0, T] . \\
& y(t) \in[0, L], \\
& \|u(t)\|_{\infty} \leq 1,
\end{aligned}
$$

In the following two particular cases, for simplicity of notation, we will not indicate the time $t$ as an independent variable of the other variables, although this is the case we are considering.

2.1. Couette Flow: Linear Velocity Profile. In the case of linear flow (with slope $m=v_{0} / L>0$ ), the velocity field of the flow is given by $v(x, y)=(y / m, 0)$. So, the dynamics of this control system are given by $F(X, u)=\left(y / m+u_{x}, u_{y}\right)$.

The Pontryagin maximum principle, [6], allows us to determine the optimal control $u^{*}=\left(u_{x}^{*}, u_{y}^{*}\right)$ by using the maximization of Pontryagin's function $H(X, P, u)$ (here, $P=$ $\left(p_{x}, p_{y}\right)$ is the adjoint variable satisfying $-\dot{P}=\nabla_{X} H(X, P, u)$, $\nabla_{X}$ being the gradient of $H$ with respect to $X$ ) almost everywhere with respect to the Lebesgue measure (from here onwards, functions are specified in this sense), together with the satisfaction of the appropriate boundary conditions. So, being

$$
H(X, P, u)=p_{x}\left(\frac{y}{m}+u_{x}\right)+\left(p_{y}+\gamma\right) u_{y},
$$

where $\gamma$ is a certain function which reflects the activity of the state constraints of the variable $y$, it follows that

$$
\begin{gathered}
-\dot{p}_{x}=0, \\
-\dot{p}_{y}=\frac{p_{x}}{m},
\end{gathered}
$$

and, thus,

$$
\begin{gathered}
p_{x}=K_{x}, \\
p_{y}=K_{y}-\frac{K_{x}}{m} t,
\end{gathered}
$$

for some constants $K_{x}, K_{y}>0$. By taking into account that the position of the particle at time $t$ is given by

$$
\begin{gathered}
x(t)=\frac{b}{m} t+\int_{0}^{t} u_{x}(\tau) d \tau+\frac{1}{m} \int_{0}^{t}(t-\tau) u_{y}(\tau) d \tau, \\
y(t)=b+\int_{0}^{t} u_{y}(\tau) d \tau,
\end{gathered}
$$

we conclude by the maximization of Pontryagin's function that $u_{x}^{*}(t)=1$ (Figure 2) and $u_{y}^{*}$ is given by the following.

(i) Consider

$$
u_{y}^{*}(t)= \begin{cases}1, & t \in\left[0, t^{*} / 2[\right. \\ -1, & \left.t \in] t^{*} / 2, t^{*}\right]\end{cases}
$$

for the case of $x_{f} \leq 2(L-b)$. By substituting in the equations of the particle's position, we conclude that the optimal time of (3) is given by

$$
t^{*}=2\left(\sqrt{(b+m)^{2}+m x_{f}}-(b+m)\right) .
$$

(ii) Consider

$$
u_{y}^{*}(t)= \begin{cases}1, & t \in\left[0, t_{1}[,\right. \\ 0, & t \in] t_{1}, t^{*}-t_{1}[, \\ -1, & \left.t \in] t^{*}-t_{1}, t^{*}\right],\end{cases}
$$




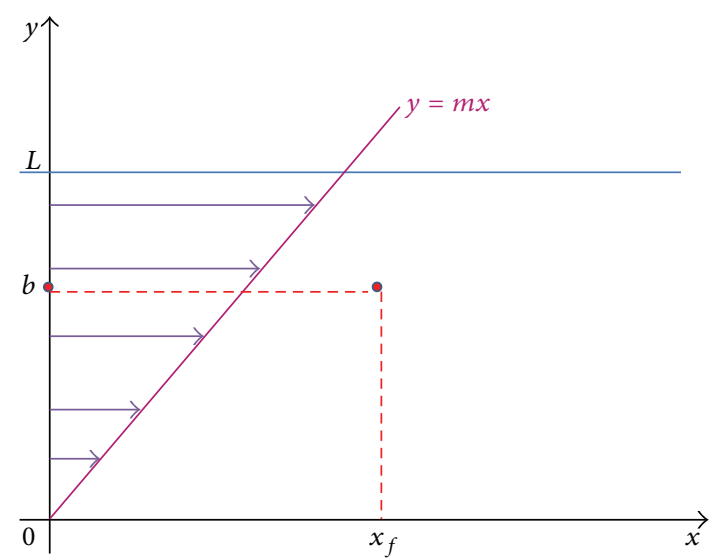

(a)

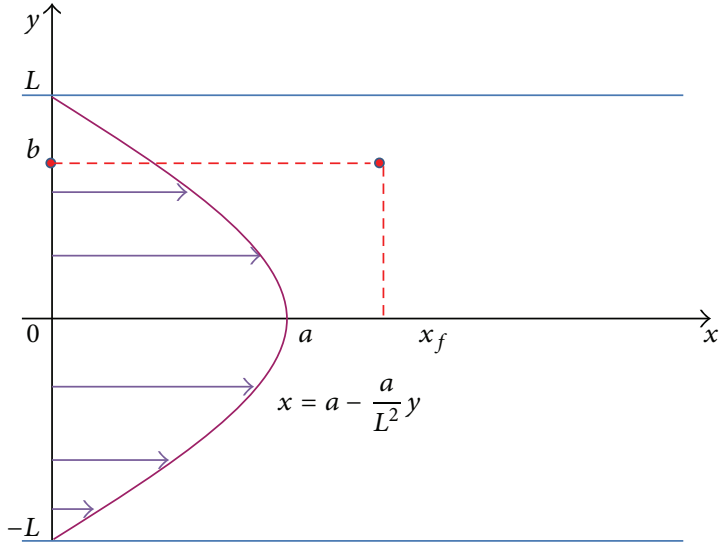

(b)

FIGURE 2: Linear (a) and quadratic velocity field (b).

for the case of $x_{f}>2(L-b)$, where

$$
t_{1}=\sqrt{(b+m)^{2}+2 m(L-b)}-(b+m),
$$

being now

$$
\begin{aligned}
t^{*}= & \left(2(b+m)^{2}-2(b+m) \sqrt{(b+m)^{2}+2 m(L-b)}\right. \\
& \left.+2 m(L-b)+m x_{f}\right) \\
& \times\left(\sqrt{(b+m)^{2}+2 m(L-b)}\right)^{-1} .
\end{aligned}
$$

The two cases in the definition of $u_{y}^{*}$ correspond to the situations in which the constraint is inactive and active, respectively.

2.2. Poiseuille Flow: Parabolic Velocity Profile. Similar arguments are applied to the case of flow with parabolic velocity flow (with vertex in $(a, 0))$. Now, the dynamics of the control system is given by

$$
F(X, u)=\left(a-\frac{a}{L^{2}} y^{2}+u_{x}, u_{y}\right),
$$

and Pontryagin's function is given by

$$
H(X, P, u)=p_{x}\left(a-\frac{a}{L^{2}} y^{2}+u_{x}\right)+\left(p_{y}+\gamma\right) u_{y} .
$$

It is easy to observe that the state constraint will be inactive along the optimal trajectory and that there is symmetry about the axis $y=0$. By using these observations in the application of the Pontryagin maximum principle, as well as the fact that the position of the particle is given by

$$
\begin{gathered}
x(t)=a t-\frac{a}{L^{2}} \int_{0}^{t} y^{2}(\tau) d \tau+\int_{0}^{t} u_{x}(\tau) d \tau, \\
y(t)=b+\int_{0}^{t} u_{y}(\tau) d \tau,
\end{gathered}
$$

we conclude that $u_{x}^{*}(t)=1$ (Figure 2 ) and that $u_{y}^{*}$ is defined by the following:

(i)

$$
u_{y}^{*}(t)= \begin{cases}-1, & t \in\left[0, t^{*} / 2[\right. \\ 1, & \left.t \in] t^{*} / 2, t^{*}\right]\end{cases}
$$

if $x_{f} \leq 2 b$, in this case, the minimum time $t^{*}$ being a root of the polynomial as follows:

$$
\begin{gathered}
t^{* 3}-6 b t^{* 2}+\left(12 b^{2}-12 L^{2}-\frac{12 L^{2}}{a}\right) t^{*} \\
+\frac{12 L^{2}}{a} x_{f}=0,
\end{gathered}
$$

(ii)

$$
u_{y}^{*}(t)= \begin{cases}-1, & t \in\left[0, t_{1}[,\right. \\ 0, & t \in] t_{1}, t^{*}-t_{1}[, \\ 1, & \left.t \in] t^{*}-t_{1}, t^{*}\right],\end{cases}
$$

if $x_{f}>2 b$, being the optimal time as follows:

$$
t^{*}=\frac{x_{f}-\left(2 a / L^{2}\right)\left((2 / 3) t_{1}^{3}-b t_{1}^{2}\right)}{a+1-\left(a / L^{2}\right)\left(b-t_{1}\right)^{2}},
$$

where $t_{1}$ is half of the value of the $t^{*}$ obtained in (17) with $x_{f}=2 b$.

\section{Conclusions and Future Work}

The case studies discussed here are very simple and their difference concerns only the profile of the velocity of the fluid. Not only the dynamics of the control system are defined by a set of ODEs, but also the conditions resulting from the application of the Pontryagin maximum principle can be easily 
solved in an explicit way. The next step consists in deriving optimality conditions in the form of a maximum principle leading to the computation of the solution to optimal control problems for which the previous simplifications cannot be exploited. This study suggests that the optimality conditions to be developed will require an adjoint variable satisfying a mixed system with ODEs and PDEs, so that the optimal control can be obtained by maximizing an appropriated Pontryagin function, coupled with appropriate boundary conditions.

\section{Acknowledgments}

The first and the second authors gratefully acknowledge the financial support of the FCT funded R\&D projects PESTOE/EEI/UI0147/2011 and PTDC/EEA-CRO/104901/2008. The work of the third author was supported, in part, by FCT through the CMUP_Centro de Matemática da Universidade do Porto.

\section{References}

[1] F. H. Clarke, Y. S. Ledyaev, R. J. Stern, and P. R. Wolenski, Nonsmooth Analysis and Control Theory, vol. 178 of Graduate Texts in Mathematics, Springer, New York, NY, USA, 1998.

[2] J.-L. Lions, Optimal Control Of Systems Governed by Partial Differential Equations, Springer, New York, NY, USA, 1971.

[3] N. Mahmoudian, J. Geisbert, and C. Woolsey, "Dynamics and control of underwater gliders I: steady motions," Tech. Rep. no. VaCAS-2007-01, Virginia Center for Autonomous Systems, 2009.

[4] N. Mahmoudian and C. Woolsey, "Dynamics and control of underwater gliders II: motion planning and control," Virginia Center for Autonomous Systems no. VaCAS-2010-02, 2010.

[5] J. Liu and H. Hu, "Biological inspiration: from carangiform fish to multi-joint robotic fish," Journal of Bionic Engineering, vol. 7, no. 1, pp. 35-48, 2010.

[6] L. Pontryagin, V. Boltyanskiy, R. Gamkrelidze, and E. Mishchenko, Mathematical Theory of Optimal Processes, Interscience, New York, NY, USA, 1962.

[7] A. V. Arutyunov, D. Y. Karamzin, and F. L. Pereira, "The maximum principle for optimal control problems with state constraints by R. V. Gamkrelidze: revisited," Journal of Optimization Theory and Applications, vol. 149, no. 3, pp. 474-493, 2011.

[8] F. Clarke, "The maximum principle in optimal control, then and now," Journal of Control and Cybernetics, vol. 34, no. 3, pp. 709722, 2005.

[9] G. K. Batchelor, An Introduction to Fluid Dynamics, Cambridge Mathematical Library, Cambridge University Press, Cambridge, UK, 1999. 


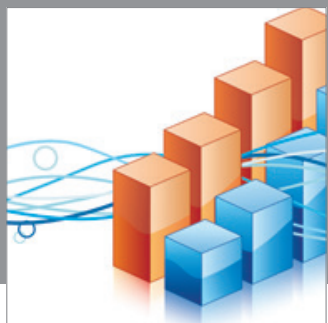

Advances in

Operations Research

mansans

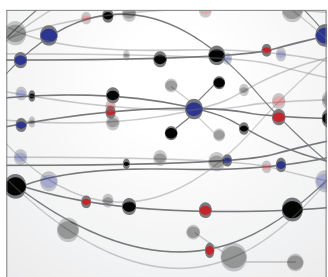

The Scientific World Journal
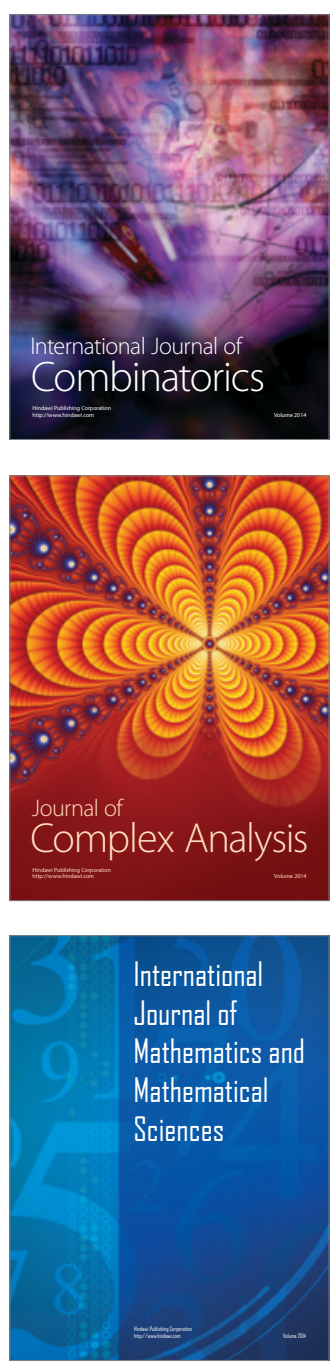
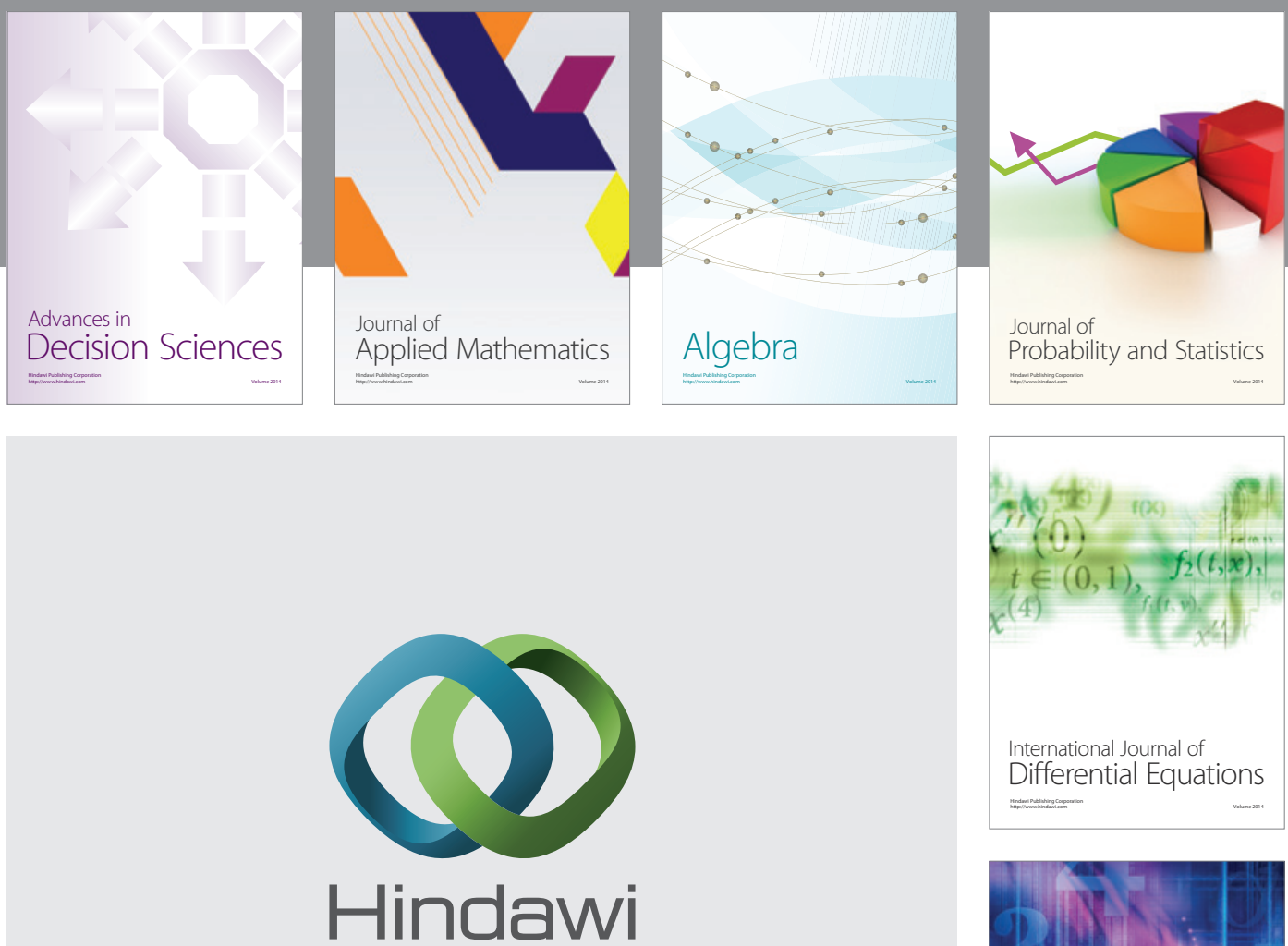

Submit your manuscripts at http://www.hindawi.com
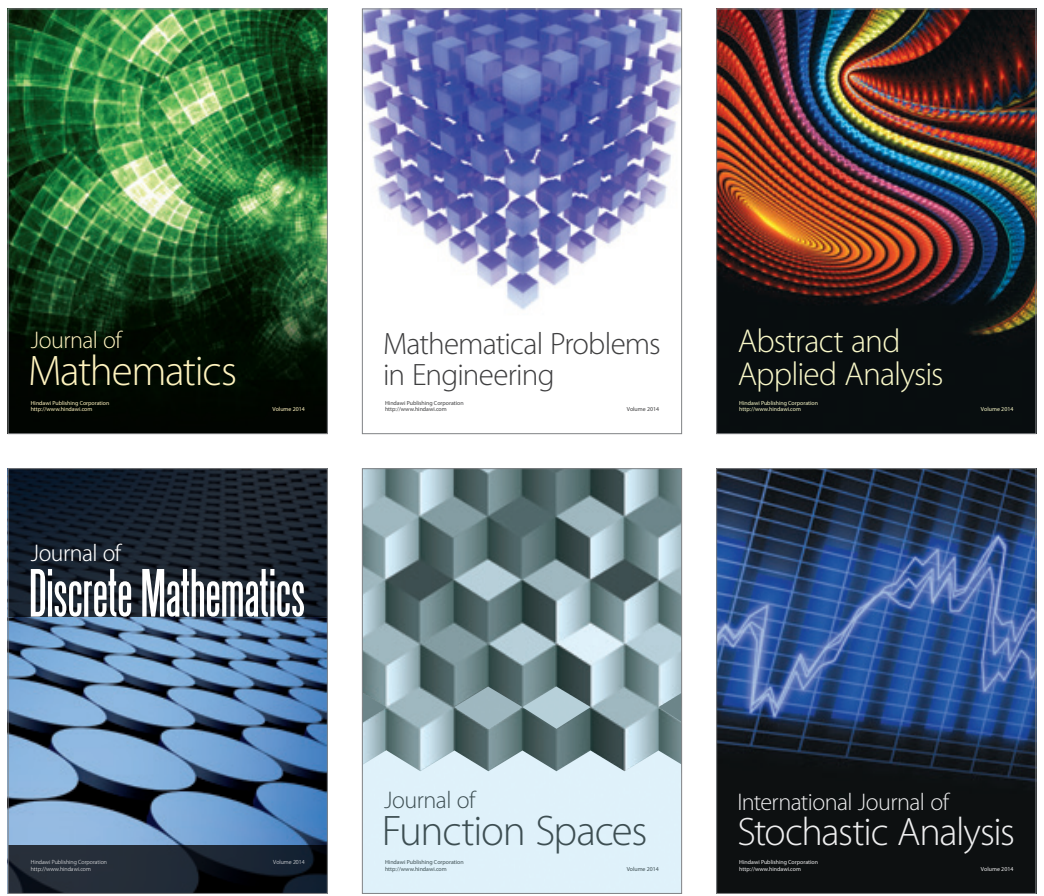

Journal of

Function Spaces

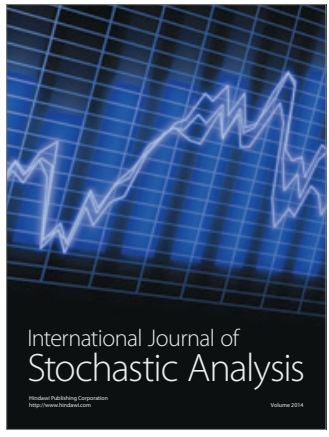

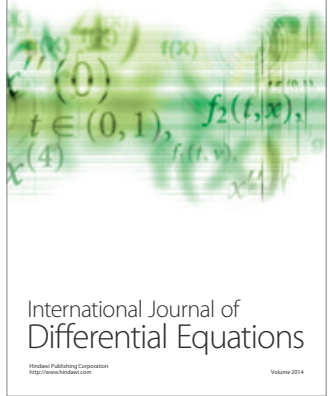
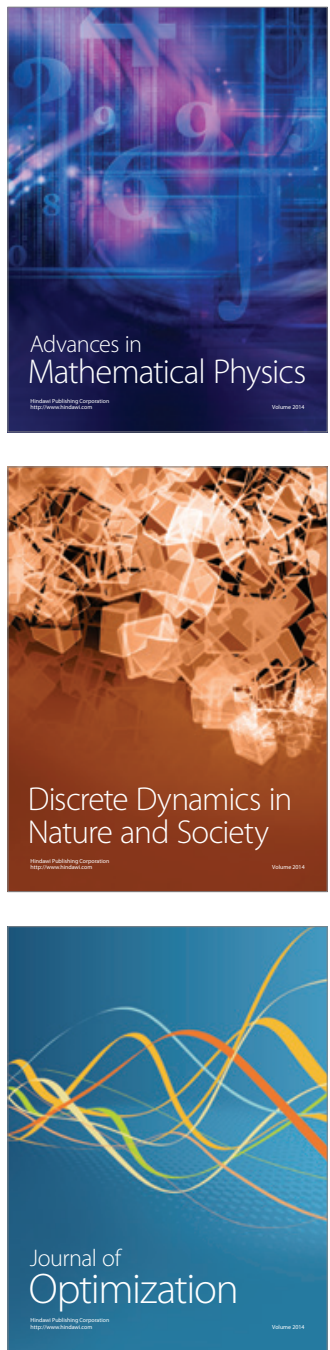